\title{
Analysis of the Efficiency of Elementary Grids of Axial Compressors Based on the Data of Flat Grid Purges
}

\author{
A.I. BOROVKOV, YU.B. GALERKIN, YU.A. POPOV, A.F. REKSTIN, O.A. SOLOVYOVA, A.A. DROZDOV, L.N. \\ MARENINA, CHERNIKOV V.A. \\ Leading Research Center "Digital Design and Modeling (Smart Design)" \\ Peter the Great St.Petersburg Polytechnic University \\ 195251, St.Petersburg, Polytechnicheskaya, 29 \\ RUSSIA
}

Abstract: - Published data on flat grid purges allow to evaluate the efficiency of blade devices with different kinematic scheme of stages and design parameters. The authors present the main provisions of the calculation program algorithm and some results of numerical analysis of the efficiency of flat grids of axial stages with different combinations of design parameters. The content of the article and the computer program are addressed to people who are improving their knowledge of gas dynamics of axial compressors. Some of the results may be of interest to specialists working professionally in this field.

Key-Words: - Mathematical simulation, axial compressor, impeller, guiding device, efficiency, loss coefficient, theoretical head, velocity triangle, bushing ratio

Received: February 13, 2020. Revised: August 23, 2020. Accepted: September 1, 2020. Published: September $12,2020$.

\author{
Legend \\ B - blade chord \\ $\mathrm{c}$ - absolute velocity (flow rate in fixed coordinate \\ system) \\ $F_{D}$ - diffusion airfoil factor \\ $\mathrm{h}_{\mathrm{w}}$ - loss of head \\ $\mathrm{t}$ - grid spacing (distance between blades) \\ $\mathrm{w}$ - relative velocity (flow rate in a rotating \\ coordinate system); \\ $\mathrm{u}$ - circumferential velocity; \\ $\alpha$ - angle between the absolute velocity and the \\ circumferential direction \\ $\beta$ - angle between the relative velocity and the \\ circumferential direction \\ $\zeta$ - loss of head coefficient in grid \\ $\varepsilon=\beta_{2}-\beta_{1}$ or $\alpha_{2}-\alpha_{1} 1-$ flow turning angle of \\ impeller or guiding device blades \\ $\varphi=\mathrm{c}_{\mathrm{z}} / \mathrm{u}=\mathrm{w}_{\mathrm{z}} / \mathrm{u}_{2}$ - flow coefficient \\ $\eta-$ polytropic efficiency \\ $\psi_{\mathrm{T}}=\left(\mathrm{w}_{\mathrm{u} 1}-\mathrm{w}_{\mathrm{u} 2}\right) / \mathrm{u}-$ loading factor \\ $\Omega=1-\left(\mathrm{W}_{\mathrm{u} 1}+\mathrm{W}_{\mathrm{u} 2}\right) / 2 \mathrm{u}$ - "theoretical" degree of \\ reaction
}

\section{Subindexes \\ 1,2 - flow parameters at the inlet and outlet of the impeller blade grid, respectively \\ GD - guiding device grid; \\ ImBS - impeller bucket system; \\ $\mathrm{u}$ - velocity projection on circumferential direction}

$\mathrm{z}$ - velocity projection on axial direction

\section{Introduction}

Published data on flat grid purges allow to evaluate the efficiency of blade devices with different kinematic scheme of stages and design parameters. An example of this kind of numerical analysis is presented in Chapter 2 of the monograph [1]. The authors present the main provisions of the calculation program algorithm and some results of numerical analysis of the efficiency of flat grids of axial stages with different combinations of design parameters. The content of the article and the computer program are addressed to people who are improving their knowledge of gas dynamics of axial compressors. Some of the results may be of interest to specialists working professionally in this field.

\section{Literature review}

The principles of axial compressor blade devices profiling based on testing of flat grids in the Russian literature were described in [2], in more detail - in [3]. For axial fans, the results of extensive research and design methods are presented in the monograph [4]. Recommendations for the design of industrial axial compressors are contained in the technical materials [5] and others. A number of specific design issues have been solved by domestic researchers and described in a number of publications, for example, 
in [6]. The works [7], [8] present the development of a software package for the optimization of size and form of axial compressors. The developed mathematical model takes into account various components of head losses in axial compressors and allows optimizing their dimensions, impeller and guiding device blades form, and provides predicted gas-dynamic characteristics of the compressor. The work [9] presents an improved model for transonic axial compressors calculation and design based on two-dimensional calculations. The model was verified based on experimental studies of a transonic axial stage and a three-stage P\&W 3S1 compressor. The work [10] presents the concept of the through flow method. In recent decades, the through flow method has become one of the most important tools for designers - a compromise between the accuracy of the flow field representation and the corresponding computational cost. The work [11] presents a program for optimizing the impeller blades of axial compressor stages based on a proprietary CFD program. The authors created an automatic algorithm for computational grid rebuilding and an optimizer that allows varying the geometry of the blade surfaces on the current lines to achieve a given pressure distribution on the blades. The work [12] presents a program for preliminary evaluation of the layout and form of axial turbomachines of transport GTE. The program is designed to analyze a variety of options for the flow part and select the optimal number of compressor stages, the meridional form of the flow path, etc. The authors note that the use of pre-design programs is an integral step in the creation of new GTE. The work [13] presents a program for preliminary design of an axial multistage compressor. The calculation and optimization in it is carried out for the center current line along the height of the compressor blades. The underlying mathematical model allows also to design supersonic compressors. However, the loss calculation is performed only for direct shock waves and the calculation algorithm does not take into account changes in flow parameters after shock wave. This slightly reduces the functionality of this program. The work [14] presents a mathematical model for calculating the characteristics of multistage axial compressors. It is an extended one-dimensional model for calculating the flow parameters on the center current line along the blade height, which the authors themselves call 1.5D. The authors present the results of verification of their model for two axial compressors. In works [15], [16] the code of the center line of an axial compressor operating in noncalculating modes is described for specialized engineering software that is developed for the analysis of the entire gas turbine engine. A generalized methodology for losses simulation on the midline of the axial compressor has been developed. A number of researchers only use CFD calculations to optimize the form of axial compressor stages. An example of such work is the publication [17].

In work [18], a three-stage axial compressor with an inlet guiding device operating with a transonic flow pattern is calculated. The influence of accounting for leaks through labyrinth seals on compressor characteristics is studied. Computer programs based on the flow organization principle and mathematical models from work [19] work well in relation to compressors with subsonic gas movement [20]-[23]. When designing blade devices of subsonic stages based on the results of plane grids purges, a diagram of nominal modes is usually used, which summarizes the experimental data of A. Howell [24]. The diagram establishes the relationship between the main design parameters of the stage at the calculated radius: the reactivity degree $\Omega$, the flow coefficient $\varphi$, and the loading factor $\psi_{\mathrm{T}}$. The relative spacing $\mathrm{t} / \mathrm{B}$ or the inverse density of the grid $\mathrm{B} / \mathrm{t}$ acts as a parameter of the functional dependence.

Following the ratios recommended by the diagram ensures that there are no gross errors in the design of blade grids - in particular, it allows to avoid too much deceleration of the flow on the rarefaction side of the blades. Generalized data on grid losses allow to supplement the recommendations of the nominal mode diagram with some quantitative relations.

It is known that axial stages are operable only at very small (in comparison, for example, with centrifugal stages) values of loss coefficients. This is due to the fact that the work supplied to the gas in the axial stages is relatively small, and the gas kinetic energy, to which the head loss is proportional, is on the contrary very significant. The influence of these factors on efficiency is determined by the following dependencies:

$\eta=1-\Delta \eta$,
$\Delta \eta=\Delta \eta_{I m}+\Delta \eta_{G D}=\frac{\varsigma_{I m}}{2 \psi_{\mathrm{T}}}\left(w_{1} / u\right)^{2}+\frac{\varsigma_{G D}}{2 \psi_{\mathrm{T}}}\left(\mathrm{c}_{2} / u\right)^{2}$.

- let's recall that the absolute velocity at the outlet of the impeller is the inlet velocity of the guiding device, and vice versa.

Continuing the comparison with centrifugal stages, we note the following:

the loading factors of the axial stages are noticeably lower: by a third, or even twice,

the flow rates in relation to the circumferential velocity $\mathrm{w} 1 / \mathrm{u}$ and $\mathrm{c} 1 / \mathrm{u}$ for axial stages with usually 
accepted flow coefficients in the design mode are close to or greater than one. For centrifugal stages of industrial compressors this ratio rarely exceeds 0.65 ... 0.70. Accordingly, the kinetic energy of the flow in the axial stages is "several times" higher.

Obtaining small loss coefficients in the axial stages is facilitated by:

favorable flow pattern in the meridional plane. The flow moves almost axially, while in the centrifugal stages it turns 90-180 degrees,

the relative elongation of the axial stages blades (the length of the blade relative to the chord) is usually noticeably greater than one, which helps to reduce the share of losses on the limiting surfaces of the body and hub,

the use of carefully calculated and purged blade profiles.

However, to obtain acceptable values of the loss coefficient, one more condition must be met. Experience shows that the axial stages in the design mode cannot be allowed to disrupt the flow on the surfaces of blades rarefaction. That is, acceptable values of loss coefficients can occur if the profile resistance is determined only by friction losses (for centrifugal stages in the design mode, flow stalls on the impeller blades, diffusers and $\mathrm{RCh}$ (return channel) - common and acceptable phenomenon.). Limiting the profile friction losses is achieved by selecting the relative spacong of the grids $\mathrm{t} / \mathrm{B}$, which is recommended to be selected within $0.7 \ldots 1.0$ at the calculated radius of the stage. At $\mathrm{t} / \mathrm{B}<0.7$, the pressure loss increases, and at $\mathrm{t} / \mathrm{B}>$ 1.0 , the loading factor decreases.

\section{Research results}

\subsection{Basic equations for calculating elementary grids} with consideration of the diffusion airfoil factor

In accordance with the S. Lieblein [25] data, the velocities ratio along the rarefaction surface of the blade should be controlled using the diffusion airfoil factor $\mathrm{F}_{\mathrm{D}}=1-\mathrm{w}_{2} / \mathrm{w}_{3} \max -$ a typical diagram of surface velocities on the blades is shown in Fig. 1. The value of $F_{D}=0.45$ at the design mode provides continuous flow at a flow rate equal to and slightly less than the calculated one.

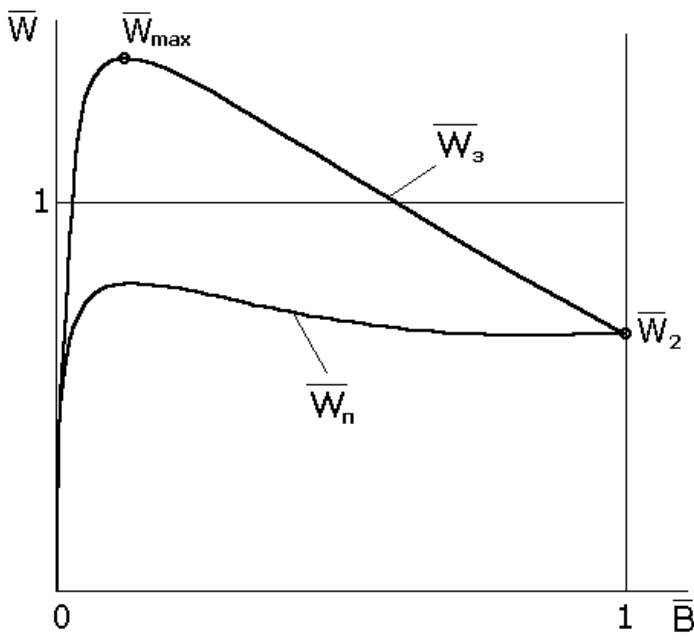

Fig. 1. Typical diagram of surface velocities on the blades of an axial impeller - design mode

Under the usually accepted condition that the flow rate component is equal in all control sections of the stage and the moves along the current surface with a constant radius, and taking into account the known relations between the reactivity degree $\Omega=1-$ (cu1+ $\mathrm{cu} 2) / 2 \mathrm{u}$ and the loading factor $\psi \mathrm{T}=(\mathrm{cu} 2-\mathrm{cu} 1) / \mathrm{u}$, the values of the circumferential projections of the absolute velocity:

$$
\begin{aligned}
& \mathrm{cu} 1 / \mathrm{u}=1-\Omega-0.5 \psi \mathrm{T} \\
& \mathrm{cu} 2 / \mathrm{u}=1-\Omega+0.5 \psi \mathrm{T} \\
& \mathrm{wu} 1 / \mathrm{u}=1-\operatorname{cu} 1 / \mathrm{u} \\
& \mathrm{wu} 2 / \mathrm{u}=1-\mathrm{cu} 2 / \mathrm{u},
\end{aligned}
$$

Taking into account the known ratio between the velocities and their axial and circumferential components, the ratio (1) is converted to the form:

$$
F_{D I m}=1-\frac{\left(\phi^{2}+\left(\Omega-\psi_{T} / 2\right)^{2}\right)^{0.5}}{\left(\phi^{2}+\left(\Omega+\psi_{T} / 2\right)^{2}\right)^{0.5}} \frac{\psi_{T} t / B}{2\left(\phi^{2}+\left(\Omega+\psi_{T} / 2\right)^{2}\right)^{0.5}}
$$

Equation (3) allows to set the value of the loading factor, which corresponds to the selected values of the reactivity degree, the flow coefficient, and the relative pitch. By choosing a certain degree of reactivity, relative pitch, and acceptable value of the diffusion airfoil factor, the dependence of loading factor on the flow coefficient of the impeller can be determined, the blade grid of which is maximally loaded, but flows continuously.

The choice of the flow coefficient, the degree of reactivity, and the calculation of the loading factor corresponding to the selected diffusion airfoil factor and relative pitch allows to determine all the components of the velocity triangles, taking into account the relations (2), for example: 


$$
\begin{array}{ll}
\alpha_{1}=\operatorname{arctg} \frac{\varphi}{c_{u 1} / u}, & \alpha_{2}=\operatorname{arctg} \frac{\varphi}{c_{u 2} / u} \\
\beta_{1}=\operatorname{arctg} \frac{\varphi}{w_{u 1} / u}, & \beta_{2}=\operatorname{arctg} \frac{\varphi}{w_{u 2} / u}
\end{array}
$$

According to S. Lieblein, the loss coefficient of grids determines the following relation (calculated mode, continuous flow):

$$
\zeta_{\mathrm{Im}}=\frac{0.014}{(t / B) \sin \beta_{2}}, \quad \zeta_{G D}=\frac{0.014}{(t / B) \sin \alpha_{1}}
$$

The value of the loss coefficients according to the formulas (1.5) obviously refers to grids where the value of the diffusion airfoil factor corresponds to the recommended value of 0.45 . With a higher load on the grids, the losses increase, and vice versa. This follows from the experimental dependence of the momentum thickness on the diffusion airfoil factor for minimum loss modes obtained by S. Lieblein. In the range $\mathrm{FD}=0-0.45$, the momentum thickness increases almost linearly, and then increases sharply.

If we assume a similar dependence $\zeta=\mathrm{f}(\mathrm{FD})$, then for different values of the diffusion airfoil factor, the profile loss coefficients can be calculated as follows:

$$
\begin{array}{ll}
\zeta_{I m}=K_{F D / m} \frac{0.014}{(t / B) \sin \beta_{2}}, \quad \zeta_{I m}=K_{F D G D} \frac{0.014}{(t / B) \sin \beta_{2}}, \\
\text { where } \\
\qquad \begin{array}{ll}
K_{F D}=0.5+1.11 F_{D} & \text { at } F_{D}=0.0 \ldots 0.45, \\
K_{F D}=1.0+97\left(F_{D}-0.45\right)^{2} & \text { at } F_{D}>0.45 .
\end{array}
\end{array}
$$

(approximation of S. Lieblein's experimental data was proposed by the authors of the program).

The expression for the diffusion airfoil factor of the guiding device will appear as:

$$
F_{D G D}=1-c_{1} / c_{2}+\frac{\psi_{T}(t / B)_{G D}}{2\left(c_{2} / u\right)} .
$$

When performing calculations, it should be assumed that the values of the main stage parameters (reactivity degree, flow and pressure coefficients) are set when profiling the impeller. According to the formula (7), the relative pitch of the guiding device can be set corresponding to the recommended value $\mathrm{FD}=0.45$ (or another selected value):

$$
t / B_{G D}=F_{D}-1+c_{1} / c_{2} \frac{2\left(c_{2} / u\right)}{\psi_{T}} .
$$

The presented computer program allows to make calculations by choosing a particular reactivity degree, the pitch ratio, and the value of the diffusion airfoil factor. Calculations are performed for a number of stages of different flow rates, i.e. the variants of stages in the specified range of calculated values of flow coefficients are compared. Below are some results of calculations for this program.

At the beginning of the design, the kinematic scheme is usually chosen - i.e., the degree of reactivity. It is assumed that with the same load of the impeller and the guiding device $(\Omega=0.5)$, the efficiency is greatest, but the greater the degree of reactivity, the greater the loading factor - all other things being equal.

The rational choice of the flow coefficient is also important - it affects the efficiency, loading factor and throughput capacity of the stage. The algorithm presented above is implemented in a computer program, which allows to study the issues of optimal design quantitatively. Of course, the reliability of the quantitative results corresponds to how universal the formulas (6) are for calculating the profile loss coefficient.

3.2 Influence of the pitch ratio of the impeller grids at different flow coefficients at the design mode

In the first series of calculations, stages with $\Omega=$ 0.5 are considered at three values of the pitch ratio of the impeller blades $\mathrm{t} / \mathrm{B}=0.7,085$, and 1.0. This range corresponds to the values recommended for the design of blade devices at the average design radius

Fig. 2 compares the data on loading factors. As follows from well-known theoretical considerations, the choice of the design flow coefficient strongly affects the loading factor. In the calculated range of flow coefficients, the loading factor changes almost 2.5 times. It should be noted, however, that the minimum value of the flow coefficient $\varphi=0.25$ is not typical for axial stages at the design radius. However, this may be the case for peripheral grids of long blades. 


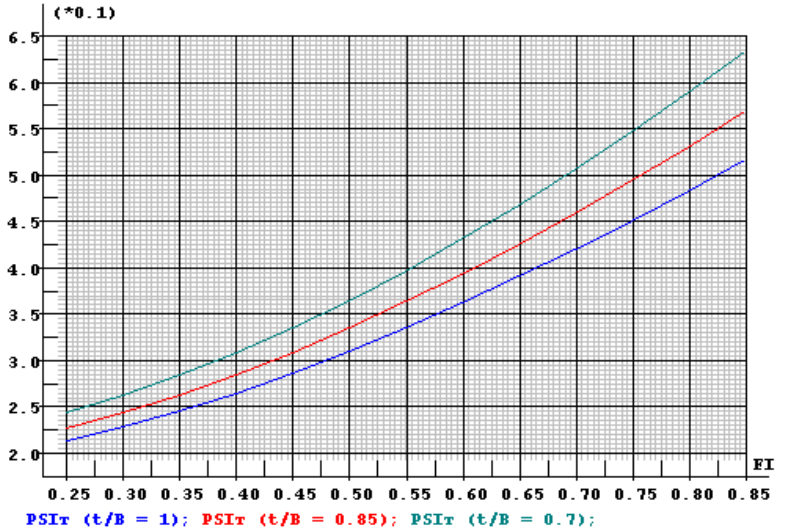

Fig. 2. Influence of the calculated flow coefficient and the pitch ratio of the impeller blades on the loading factor, $\Omega=0.5, \mathrm{FD}=0.45$.

Pitch ratio in the range of $\mathrm{t} / \mathrm{B}=0.7-1.0$ is strong enough to affect the head - within the range of 15 $22 \%$ in the practically interesting range of the design flow coefficients. However, head increase leads to decrease in efficiency, Fig. 3:

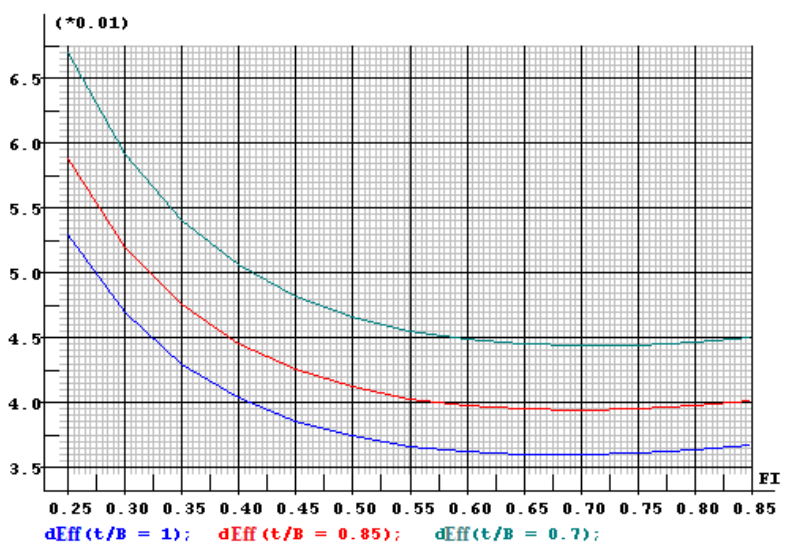

Fig. 3. Effect of the design flow coefficient and pitch ratio on the loss of efficiency due to profile losses on the impeller and guiding device blades, $\Omega=0.5$, FD $=0.45$.

Let's note the rapidly increasing losses at design flow coefficients less than 0.50 and the tendency to increase losses at $\varphi>0.70$. In the latter case, the increasing kinetic energy of the flow at the inlet to the blade devices is "guilty" - Fig. 4.

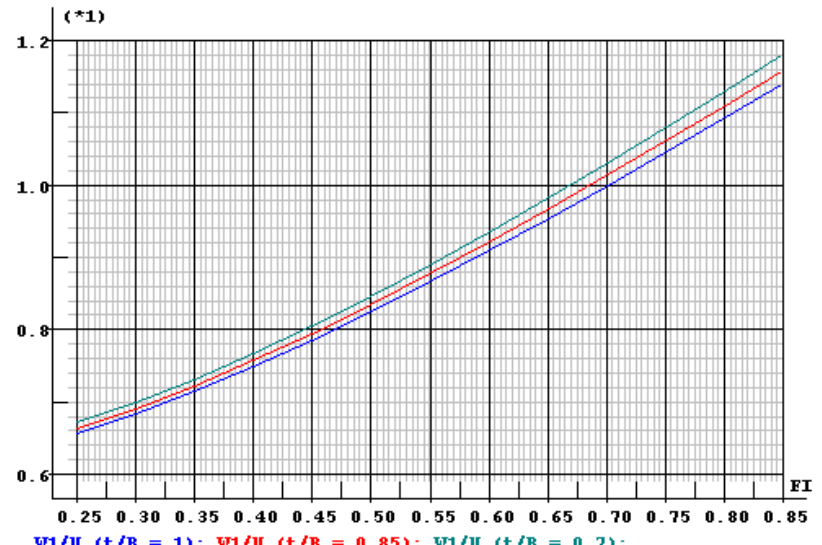

Fig. 4. Influence of the design flow coefficient and pitch ratio on the dimensionless velocity $\mathrm{w} 1 / \mathrm{u}$ at the inlet to the grid, $\Omega=0.5, \mathrm{FD}=0.45$.

It is obvious that for small flow coefficients, losses are more determined by increasing loss coefficients. The latter is due to a stronger deceleration of the flow in grids with small blade angles Fig. 5, 6 .

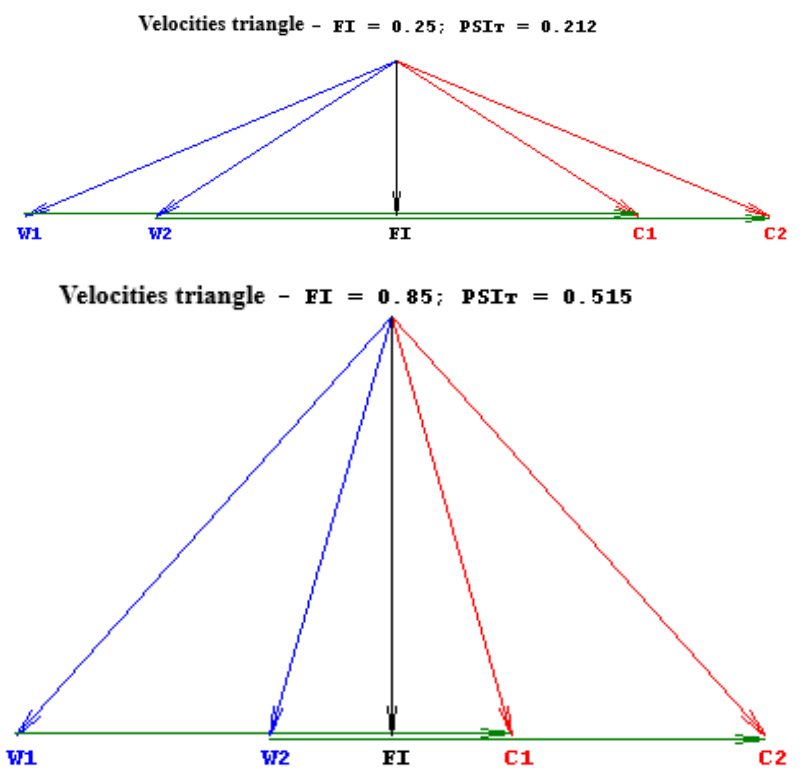

Fig. 5. Velocities triangles for different design flow coefficients, $\Omega=0.5, \mathrm{FD}=0.45, \mathrm{t} / \mathrm{B}=1.0$. 


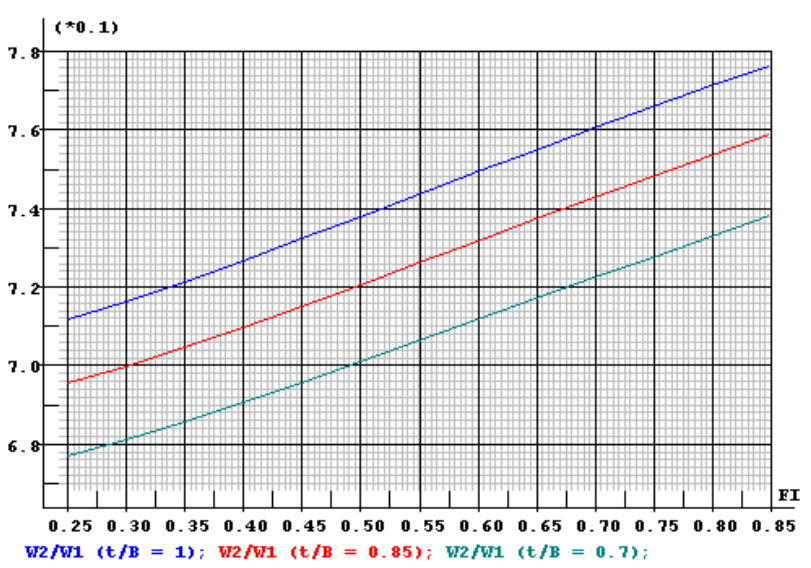

Fig. 6. Influence of the design flow coefficient and pitch ratio on the velocities ratio $\mathrm{w} 2 / \mathrm{w} 1, \Omega=0.5, \mathrm{FD}$ $=0.45$.

\subsection{Influence of the reactivity degree}

In domestic practice, it is assumed that the stages with $\Omega=0.7$ do not have a flow swirl at the inlet to the impeller [26]. In fact, for a given $\mathrm{FD}=0.45$ in the range of design flow coefficients $0.25-0.85$, the stages with $\Omega=0.7$ have a positive swirl. The positive swirl increases as the flow coefficient decreases.

The design flow coefficients corresponding to zero swirl depending on the reactivity degree are shown in the table 1 (at $\Omega=0.85$, the swirl is negative for all flow coefficients):

Table 1. Flow coefficients corresponding to zero swirl of the flow at the impeller inlet $(F D=0.45, t / B$ $=1.0)$

\begin{tabular}{|l|l|l|l|l|}
\hline$\Omega$ & 0.750 & 0.775 & 0.800 & 0.825 \\
\hline $\begin{array}{l}\varphi d e s \\
(\text { cul=0). }\end{array}$ & 0.825 & 0.745 & 0.575 & 0.445 \\
\hline
\end{tabular}

The change in the loading factor and efficiency losses depending on the design flow coefficient at three values of the reactivity degree is shown in Fig. 7. The reactivity value 0.785 intermediate between $\Omega$ $=0.5$ and 1.0 is chosen so that the zero swirl corresponds to a moderate flow coefficient $\varphi=0.685$.

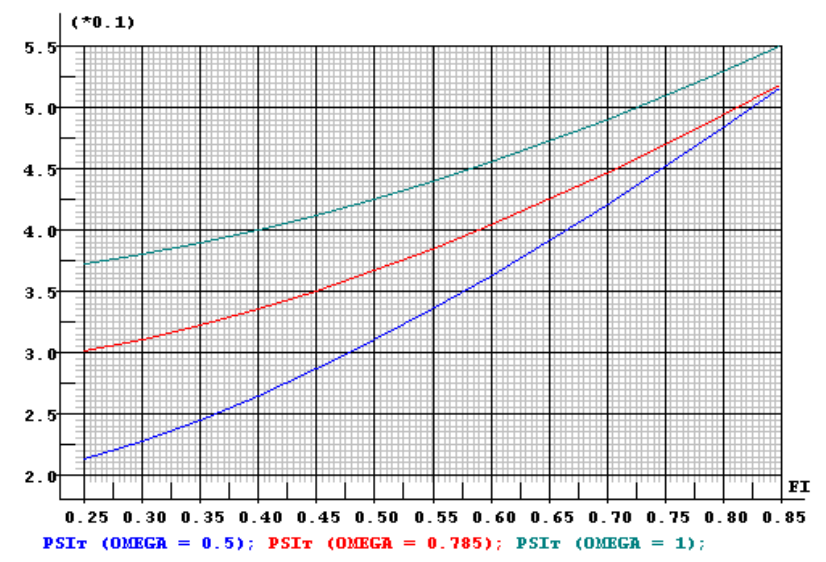

Fig. 7. Influence of the design flow coefficient and reactivity degree on the loading factor, $\mathrm{t} / \mathrm{B}=1.0, \mathrm{FD}$ $=0.45$.

The data in figure 7 demonstrate the well-known advantage of stages with a higher degree of reactivity along the head. However, for very large design flow coefficients, the stages with $\Omega=0.5$ and 0.785 differ little in the head coefficients.

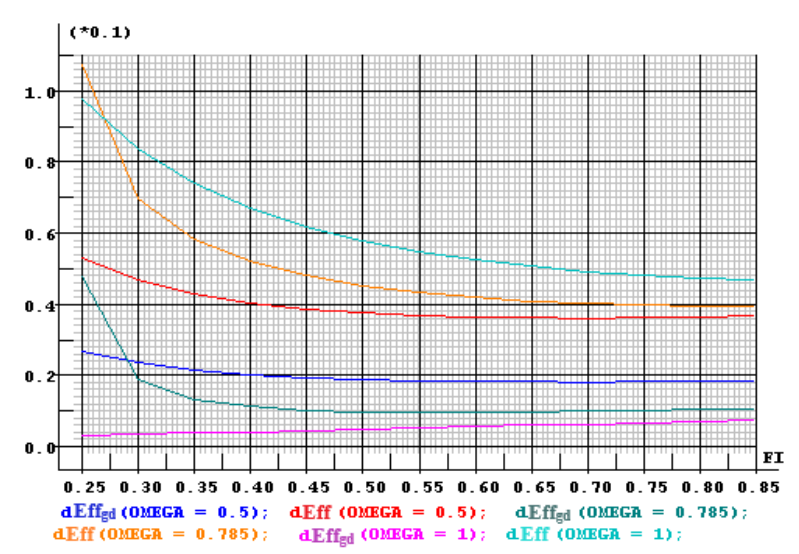

Fig. 8. Influence of the design flow coefficient and reactivity degree on efficiency losses in the guiding device and stage, $\mathrm{t} / \mathrm{B}=1.0, \mathrm{FD}=0.45$.

Stages with $\Omega=0.5$ are more efficient for all design flow coefficients. As noted earlier, with low flow coefficients, the efficiency of the stages decreases. At the same time, the difference in the efficiency of stages with different reactivity increases.

Attention is drawn to the sharp increase in efficiency losses for stages with $\Omega=0.785$ with flow coefficients less than 0.35 . With ultra-low flow coefficients less than 0.27 , such stages are even less efficient than those with $\Omega=1.0$. The data in Fig. 8 shows that this is due to an increase in losses in the guiding devices. The reason for this phenomenon is discussed below.

\subsection{Load of guiding devices grids at low design flow coefficients}

For stages with a reactivity of less than 0.5 , the pressure increment in the guiding device is less than in the impeller. With large design flow coefficients, the deceleration and flow rotation angle are small. The set value of the diffusion airfoil factor $F D=0.45$ is provided when the pitch ratio of the vanes is less than in the impeller, which helps to reduce losses.

However, the situation changes for stages with $\Omega$ $>0.5$ designed for small flow coefficients. The kinetic energy of the flow in the guiding devices is still less than in the impellers. However, the analysis of the flow kinematics in the guiding devices of 
stages with different reactivity indicates the specifics of stages with $\Omega>0.5$.

When the design flow coefficient of the stages with $\Omega=0.5$ decreases, a slight increase in deceleration and a decrease in the rotation angle occur in the same rotating and stationary blade devices of the stage. At a given $\mathrm{t} / \mathrm{B}_{\operatorname{Im}}=1.0$ in the range $\varphi$ from 0.85 to 0.25 , the $\mathrm{c} 1 / \mathrm{c} 2$ velocities ratio decreases from 0.778 to 0.714 , and the rotation angle from 25.5 to $9.9 \mathrm{deg}$. Thus, the loading factor for each $\varphi$ is chosen based on the given pitch ratio of the impeller blades (and the diffusion airfoil factor $\mathrm{FD}=$ $0.45)$, in all cases $t / \mathrm{B}_{\operatorname{Im}}=\mathrm{t} / \mathrm{B}_{\mathrm{GD}}=1.0$.

For stages with $\Omega=1.0$, there can be no deceleration of the flow in the guiding device by definition. However, for a given $\mathrm{t} / \mathrm{BIm}=1.0$ in the range of $\varphi$ from 0.85 to 0.25 , the rotation angle in the guiding device increases from 35.4 to 72.6 degrees. At a given diffusion airfoil factor $\mathrm{FD}=0.45$, the guiding device blades handle the desired rotation angle at $\mathrm{t} / \mathrm{BGD} \leq \geq 1.0$, depending on the design flow coefficient.

Oddly enough, the stages with a reactivity intermediate between 0.5 and 1.0 are in the worst position in terms of the load of the guiding device grids. For example, for a stage with $\Omega=0.775$ in the same range of design flow coefficients, the rotation angle increases slightly - from 31.6 to 36.7 degrees, but the flow deceleration varies within 0.863-0.687.

The velocities triangles of the stages with reactivity $\Omega=0.5,0.775,1.0$ with a flow coefficient of 0.25 and the same diffusion airfoil factor FD = 0.45 of all grids are shown in Fig. 9. For a stage with $\Omega=0.775$, the combination of a large rotation angle and a significant deceleration requires the use of a very thick grid to prevent the FD from growing beyond the set value.

The need to use thick grids at significant rotation angles, especially in combination with slowing down the flow, is illustrated by the calculation data shown in Fig. 10. If we proceed from the recommendations not to apply $\mathrm{t} / \mathrm{B}$ values less than 0.7 at the design radius, it is clear that the stages with reactivity of 0.6 and 0.9 cannot be used for design flow coefficients less than 0.33 . For a stage with $\Omega=0.775$, the maximum permissible value of the design flow coefficient is even greater: $\varphi$ des $\min =0.45$.

The need to use very thick grids of guiding devices for stages with $\Omega>0.5$ explains the increase in efficiency losses, which is shown in Fig. 8 above. It can be argued that in reality, the decrease in the efficiency of "super-thick" grids is even more significant than according to the results of the presented calculations. For example, for a stage with $\Omega=0.775$ (Fig. 10) with a design flow coefficient of
0.25 , the pitch ration in the guiding device should be equal to 0.15 . It is clear that a real grid with solid blades does not have a cross-section at all. Obviously, the formula (5) used to estimate the loss coefficient cannot be applied beyond reasonable values of the pitch ratio. The dependence of the minimum allowable design flow coefficient on the degree of reactivity calculated by the authors is shown in Fig. 11. It refers to the stages with $\mathrm{FD} \mathrm{Im}=\mathrm{FD} \mathrm{GD}=0.45$, $\mathrm{t} / \mathrm{B} \operatorname{Im}=1.0, \mathrm{t} / \mathrm{B} \mathrm{GD}=0.70$.
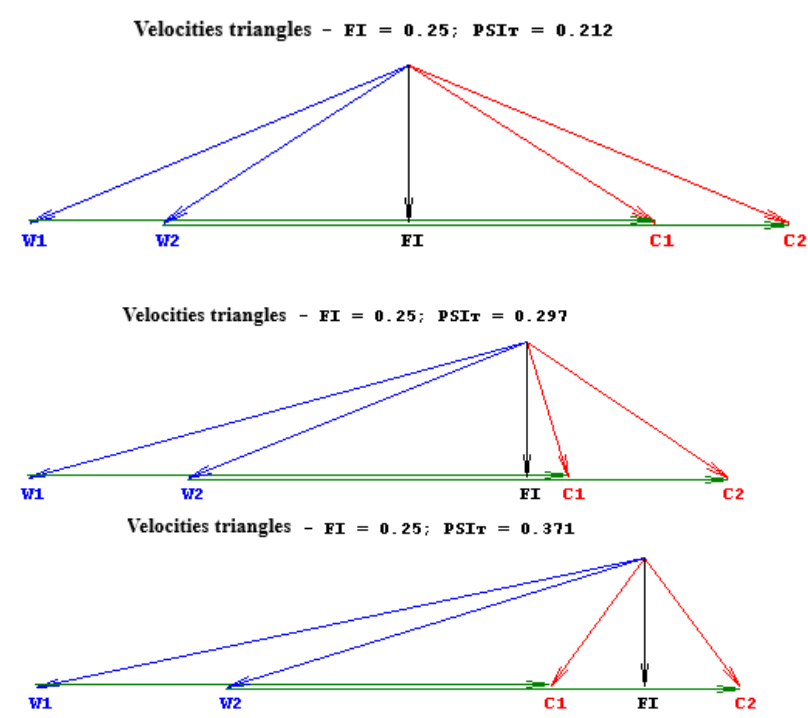

Fig. 9. The velocities triangles of the stages with reactivity $\Omega=0.5,0.775,1.0$ with a flow coefficient of 0.25 and the same diffusion airfoil factor FD $=$ 0.45 .

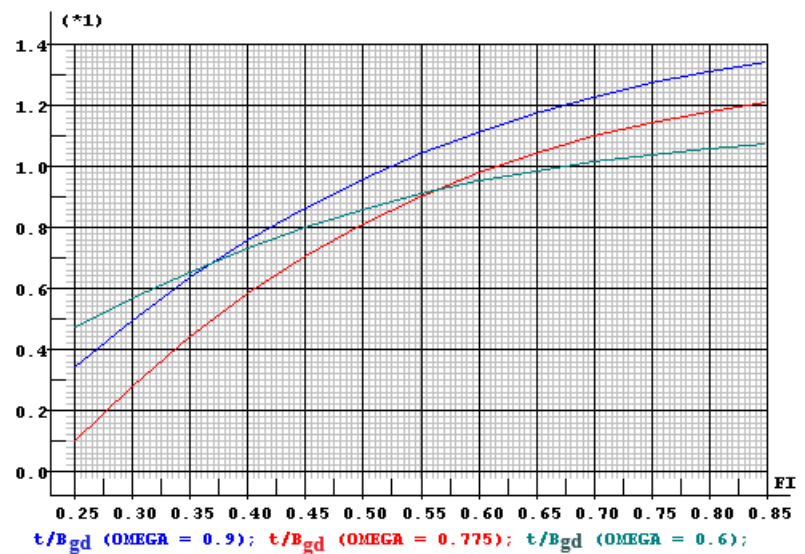

Fig. 10 Values of pitch ratio of guiding devices blades for stages with different reactivity. FD $\mathrm{Im}=$ $\mathrm{FD}$ GD $=0.45, \mathrm{t} / \mathrm{B} \mathrm{Im}=1.0$. 


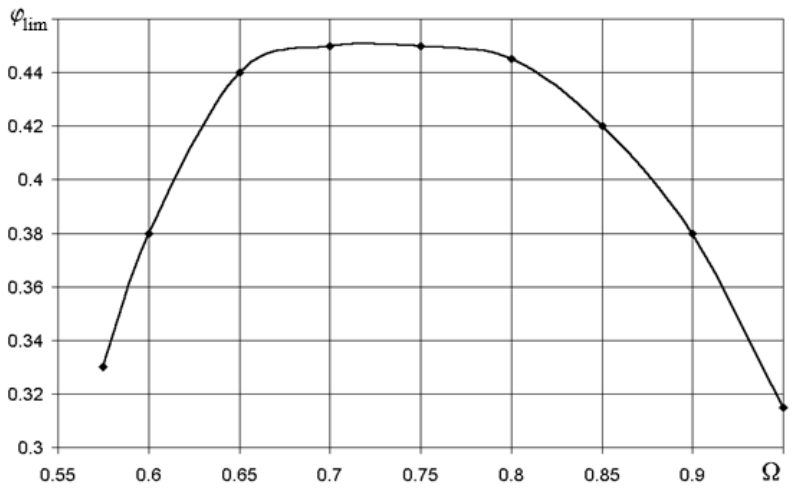

Fig. 11. Dependence of the minimum allowable design flow coefficient on the degree of reactivity. Stages with FD Im $=$ FD GD $=0.45, \mathrm{t} / \mathrm{B} \operatorname{Im}=1.0, \mathrm{t} / \mathrm{B}$ $\mathrm{GD}=0.70$.

\section{Discussion of the obtained results}

4.1 Efficiency and limits of the use of stages with a fixed pitch ratio of the guiding devices grids

Above, we considered stages with a given value of the diffusion airfoil factor of grids of impellers and guiding devices. In this case, the pitch ratio of the impeller grid was set, and the corresponding value of the loading factor was found. The pitch ratio corresponding to the specified FD was selected for the grids of the guiding devices.

Excessively thick grids of guiding devices with small design flow coefficients negatively affect the efficiency, and in extreme cases make the operation of the grid almost impossible. The developed computer program allows to perform analysis under a different condition for the guiding device - for a given value of the pitch ratio, the corresponding value of the diffusion airfoil factor is calculated.

The efficiency analysis for a fixed value of the blade pitch ratio of the guiding device blades is performed for stages with a reactivity of 0.785 , which lies in the most "problematic" zone. The calculations used the values FDIm $=0.45$ and $\mathrm{t} / \mathrm{BIm}=1.0$ for the impellers. For guiding devices, the minimum recommended value of the pitch ration $\mathrm{t} / \mathrm{BGD}=0.70$ is accepted. The calculation results are shown in Fig. 12.

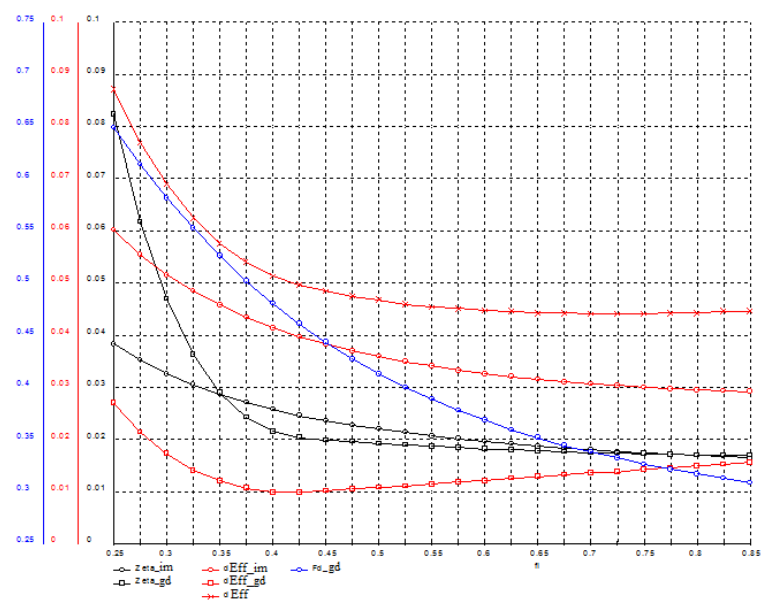

Fig. 12. Analysis of the stages efficiency at: $\Omega=0.785, \mathrm{FDIm}=0.45, \mathrm{t} / \mathrm{BIm}=1.0, \mathrm{t} / \mathrm{BGD}=0.70$.

The data in Figure 12 shows the following. With the minimum of the considered design flow coefficients of 0.25 , the diffusion airfoil factor of the guiding device blades increases to a dangerous value of 0.65 . In this case, it is likely to develop a breakdown on the back surface of the blades. The purge data used indicates a sharp increase in the loss coefficient. It increases fourfold compared to the value at the recommended $\mathrm{F}_{\mathrm{D}}=0.45$.

However, the efficiency losses in the guiding device are not too great due to the low kinetic energy of the flow. It is probably more dangerous for stages with small design flow coefficients to operate at positive headed angles, when the diffusion airfoil factor in the guiding device grid generally goes beyond the dangerous limits.

Comparison with similar stages that have a fixed diffusion airfoil factor in the guiding device shows that with a fixed pitch ration $\mathrm{t} / \mathrm{BGD}=0.70$, the losses are $0.5 \%$ greater with calculated flow coefficients $\varphi$ $=0.5$ or more. With the minimum considered $\varphi=$ 0.25 , a fixed pitch ratio provides a higher efficiency of $2.5 \%$. Here we recall once again that the calculation for a fixed diffusion airfoil factor becomes purely formal when the pitch ration of the grids is very small. For orientation, we recall that the mentioned Howell diagram of nominal modes operates with a range of pitch ratios of experimentally studied grids from 0.40 to 2.0 .

The data in Figure 12 show that stages with a reactivity of $\Omega=0.785$ are unlikely to be used for design flow coefficients less than 0.45 . When choosing the degree of reactivity closer to the values 0.5 and 1.0, the situation is improving. Stages with reactivity of 0.5 do not have any restrictions on the load of the guiding devices grids. 


\subsection{Design modes diagram and nominal modes diagram}

The results of calculations of a number of series of stages with a degree of reactivity from 0.5 to 1.0 and with pitch ratio of $0.4-2.0$ are processed by the authors in the same coordinates as the well-known A. Howell diagram of nominal modes. These are the dependences of the loading factors and flow coefficients $\psi \mathrm{T} / \varphi$ on the ratio of the reactivity degree and the flow coefficient $\Omega / \varphi$ for different $t / B$. In Fig. 13 , the diagram of the design modes with $\mathrm{FD}=0.45$ of the impeller blade devices is compared with the Howell diagram of nominal modes.

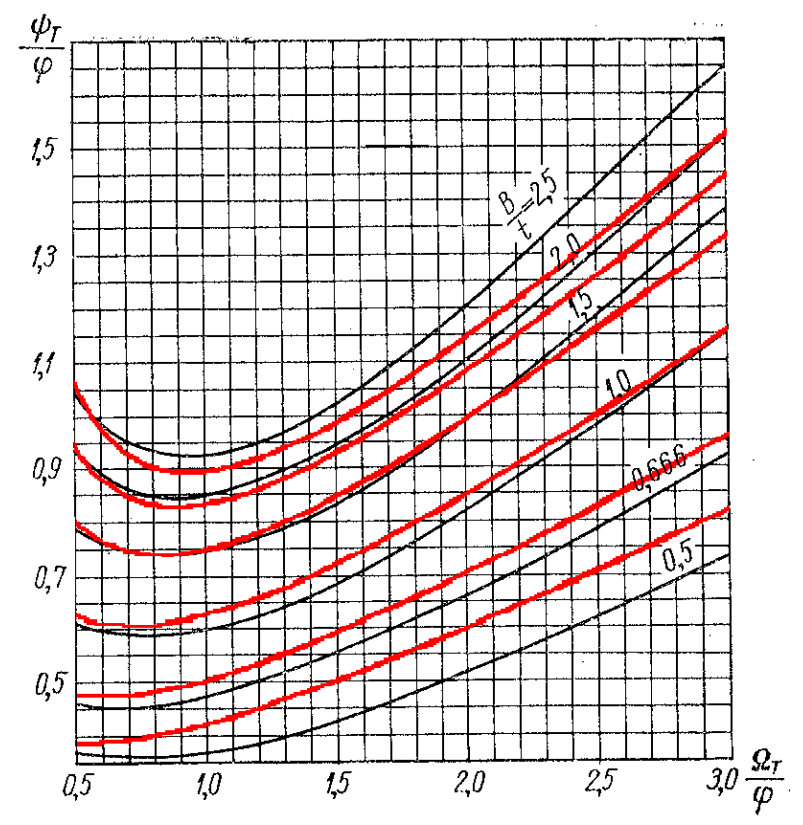

Fig. 13. Diagram of the design modes of the impeller blade devices with a given diffusion airfoil factor FD $\mathrm{Im}=0.45$ - red lines.

Howell diagram of nominal modes - black lines.

As it is known, the Howell diagram describes the so-called nominal modes. These are modes in which the angle of flow rotation is $80 \%$ of the maximum for this grid. The design modes in the diagrams below correspond to the specified diffusion airfoil factor FD $=0.45$. It is clear that with the "ideologically" identical (modes with small losses and sufficiently remote from the flow stall mode are selected), but quantitatively different approach, the diagrams are qualitatively similar, but quantitatively different.

Comparison of the diagrams shows that in the most recommended range of pitch ratios $\mathrm{t} / \mathrm{B}=0.5$ 1.0 , the calculated modes with $\mathrm{FD}=0.45$ and the Howell nominal modes are very close. However, the thick lattices with $\mathrm{t} / \mathrm{B}=0.4$ by Howell are clearly overloaded, and the sparse ones at $\mathrm{t} / \mathrm{B}=1.5-2.0-$ under loaded.
It can be assumed that the use of calculation mode diagram shown in Fig. 13 is preferable than using the traditional Howell nominal mode diagram. The diagram of design modes is based on a clearer and more reasonable physical model. When using the recommended design mode diagram to select the grid parameters, it is guaranteed to obtain the best ratios for the impeller grid (of course, within the accepted analysis scheme).

The question of the grids of guiding devices for stages with $\Omega>0.5$ remains open. As shown above, with small calculated flow coefficients, the grids of the guiding devices may be overloaded at such stages. The same problem will arise when using the Howell nominal modes diagram, since both diagrams relate the parameters of the impeller grids, but not the guiding device. The acceptable parameters of the guide arrays can be specified by calculations based on the computer program presented by the authors.

\section{Conclusion}

The presented computer program allows to analyze the gas dynamic parameters of elementary blade grids of axial compressor stages depending on the initial design parameters selected by the design engineer:

- degree of reactivity,

- flow coefficient,

- impeller pitch ratio,

- impeller diffusion airfoil factor,

- diffusion airfoil factor or pitch ratio of the guiding device grid.

The reliability of the calculated data on profile losses is determined by the degree of universality of empirical formulas for loss coefficients borrowed from foreign sources. However, according to the authors, the nature of the influence of design parameters demonstrated by the above calculations is quite reliable. For example, practice confirms head increase and decrease in efficiency with an increase in the reactivity degree from 0.5 to 1.0 .

Little known is the fact that the presented calculations result in a higher load of the stages guiding devices with an intermediate degree of reactivity between 0.5 and 1.0 at low flow coefficients. This is due to a combination of large angles of flow rotation and its strong deceleration. Stages with reactivity $\Omega=0.5$ are promising as lowflow ones.

By analogy with the well-known Howell diagram of nominal modes, the authors propose a diagram of design modes, which is characterized by a more reasonable choice of the minimum loss mode by setting the diffusion airfoil factor. 
When using the design modes diagram (as well as the Howell diagram) to select the optimal parameters of the impeller grid, the parameters of the guiding device grids should also be analyzed if the reactivity degree is $>0.5$. The computer program presented by the authors can be used for this purpose.

With medium and large design flow coefficients, the estimation of the level of profile losses seems to be underestimated. It can be assumed that when calculating profile losses over the entire height of the blades, their average value will be greater. Taking into account the losses on the limiting surfaces, the calculated efficiency may be closer to the actual efficiency of the axial compressor stages. Analysis of the effectiveness of spatial blade devices is included in the future plans of the authors.

In the future, the presented program and calculation methods are planned to be included in the programs for optimal gas-dynamic design and calculation of the multistage axial compressors characteristics. Verification of mathematical models will be carried out as new experimental data become available.

\section{Acknowledgment}

This work was financially supported by the Ministry of Science and Higher Education of the Russian Federation in the framework of the Federal Program "Research and Development in Priority Directions for the Development of the Scientific and Technological Complex of Russia for 2014-2020". Agreement on the provision of a grant in the form of subsidy No. 075-15-2019-1710 dated December 2, 2019 (internal number 05.608.21.0275). Unique identifier: RFMEFI60819X0275.

\section{References:}

[1]N.A. Cumpsty, Compressor Aerodynamics. Longman Scientific and Technical, 1989, pp. 509

[2] Yu.S. Podobuev, K.P. Seleznev, Theory and calculation of axial and centrifugal compressors Yu.S. Podobuev, K.P. Seleznev, M., L.: Mashgiz, 1957, pp. 320

[3] K.V. Kholshevnikov, Theory and calculation of aviation blade machines K.V. Kholshevnikov, Moscow: Mechanical Engineering, 1986, pp. 432

[4]I.V. Brusilovsky, Aerodynamic calculation of axial fans I.V. Brusilovsky, Moscow: Mechanical Engineering, 1986, pp. 284

[5] Method of aerodynamic calculation of the flow part of an axial compressor for stationary installations. RTM 24.020.17-73, M.: МТТЭМ, 1975, pp. 204
[6] V.V. Semov, Experimental study of a type K-3 compressor stage (K-50-3) with a small blades elongation, EMS, No. 10, 1968, pp. 30-34.

[7]M. Banjac, M.V. Petrovic, Development of method and computer program for multistage axial compressor design: part I - mean line design and example cases, Proceedings of ASME Turbo Expo 2018 Turbomachinery Technical Conference and Exposition GT2018 June 11-15, 2018, Oslo, Norway

[8] M. Banjac, M. V. Petrovic, Development of method and computer program for multistage axial compressor design: part II - twodimensional design and validation using CFD. Proceedings of ASME Turbo Expo 2018 Turbomachinery Technical Conference and Exposition GT2018 June 11-15, 2018, Oslo, Norway

[9]T. Li, Y. Wu, H. Ouyang, X. Qiang. Axial compressor performance prediction using improved streamline curvature approach, Proceedings of ASME Turbo Expo 2018 Turbomachinery Technical Conference and Exposition GT2018 June 11-15, 2018, Oslo, Norway

[10] Chen Yang, $\mathrm{Hu} \mathrm{Wu}$, Jinguang Yang and Michele Ferlauto. Time-marching throughflow analysis of multistage axial compressors based on a novel inviscid blade force model, Journal of aerospace engineering, 2019, DOI:10.1177/0954410019840588

[11] Chen Yang, $\mathrm{Hu} \mathrm{Wu}$, Yan Liang. A Novel Three-Dimensional Inverse Method for Axial Compressor Blade Surface Design, Arabian Journal for Science and Engineering

[12] Ioannis Kolias, Alexios Alexiou, Nikolaos Aretakis, Konstantinos Mathioudakis. Direct integration of axial turbomachinery preliminary aerodynamic design calculations in engine performance component models, Proceedings of ASME Turbo Expo 2018 Turbomachinery Technical Conference and Exposition GT2018 June 11-15, 2018, Oslo, Norway

[13] Youwei He, Jinju Sun, Peng Song, Xuesong Wang, Da Xu. Development of a multi-objective preliminary design optimization approach for axial flow compressors, Proceedings of ASME Turbo Expo 2018 Turbomachinery Technical Conference and Exposition GT2018 June 11-15, 2018, Oslo, Norway

[14] Olivier Adam, Olivier Léonard, A quasi-one dimensional model for axial compressors

[15] John Kidikian, Marcelo Reggio. Off-design prediction of transonic axial compressors, part 1: mean-line code and tuning factors. Proceedings of 
ASME Turbo Expo 2018. June 11-15, 2018, Oslo, Norway

[16] John Kidikian, Marcelo Reggio. Off-design prediction of transonic axial compressors, part 2: generalized mean-line loss modelling methodology. Proceedings of ASME Turbo Expo 2018. June 11-15, 2018, Oslo, Norway

[17] Xuesong Wang; Jinju Sun; Peng Song; Youwei $\mathrm{He}$; $\mathrm{Da} \mathrm{Xu}$ Three-dimensional blade shape optimization for a transonic axial flow compressor through incorporating surrogate model and sequental sampling, Proceedings of ASME Turbo Expo 2018 Turbomachinery Technical Conference and Exposition GT2018 June 11-15, 2018, Oslo, Norway

[18] Sangjo Kima, Kuisoon Kima, Changmin Sonb. Three-dimensional unsteady simulation of a multistage axial compressor with labyrinth seals and its effects on overall performance and flow characteristics, Aerospace Science and Technology, 86, 2019, pp. 11-693

[19] A.P. Komarov, Research of flat compressor grids, Blade machines and jet devices, Collection of articles, Issue 2. Moscow: Mechanical Egineering 1967, pp. 67-110.

[20] Yuri Galerkin, Yuri Popov, Optimal primary design of industrial axial compressor flow path, International Conference on Compressors and their Systems. London. City University, UK, 2009, pp. 319-329.

[21] Yu.B. Galerkin, Yu.A. Popov, The analysis of spatial flow in the "inhomogeneous" axial compressor stages, Compressor equipment and pneumatics, 2006, No. 4

[22] Yu.B. Galerkin, Yu.A. Popov, Analysis of the efficiency of spatial blade grids of axial compressors based on flat grids blowdown data, Compressor equipment and pneumatics, 2005, No. 3

[23] Yu.B. Galerkin, Yu.A. Popov, Computational study of the characteristics of axial compressor stages, Compressor equipment and Pneumatics, 2005, No. 5.

[24] A.R. Howell, Fluid dynamics of axial compressors. Proc. I Mech. E. 153: 1945, 441, 82

[25] S. Lieblein, Experimental flow in 2D cascades, Chapter V1 of The Aerodynamic Design of Axial Flow Compressor. Reprinted NASA SP 36 in 1965.

[26] K.P. Seleznev, Yu.b. Galerkin, etc. Theory and calculation of turbine superchargers, Mechanic engineering, 1986

\section{Contribution of individual authors to the creation of a scientific article (ghostwriting policy)}

\author{
A.I. Borovkov, Yu.B. Galerkin, V.A. Chernikov have \\ organized the researches \\ Yu.A. Popov, O.A. Solovieva, A.A. Drozdov were \\ developed 2D mathematical model \\ A.F. Rekstin, L.N. Marenina were provide numerical \\ resarches \\ Follow: www.wseas.org/multimedia/contributor- \\ role-instruction.pdf
}

This work was financially supported by the Ministry of Science and Higher Education of the Russian Federation in the framework of the Federal Program "Research and Development in Priority Directions for the Development of the Scientific and Technological Complex of Russia for 2014-2020". Agreement on the provision of a grant in the form of subsidy No. 075-15-2019-1710 dated December 2, 2019 (internal number 05.608.21.0275). Unique identifier: RFMEFI60819X0275.

\section{Creative Commons Attribution License 4.0 (Attribution 4.0 International, CC BY 4.0)}

This article is published under the terms of the Creative Commons Attribution License 4.0 https://creativecommons.org/licenses/by/4.0/deed.en_US 